\title{
Control robusto QFT para estabilización en cuadricóptero utilizando técnicas de prototipado rápido
}

QFT robust control for stabilization in quadrotor based on rapid prototyping techniques

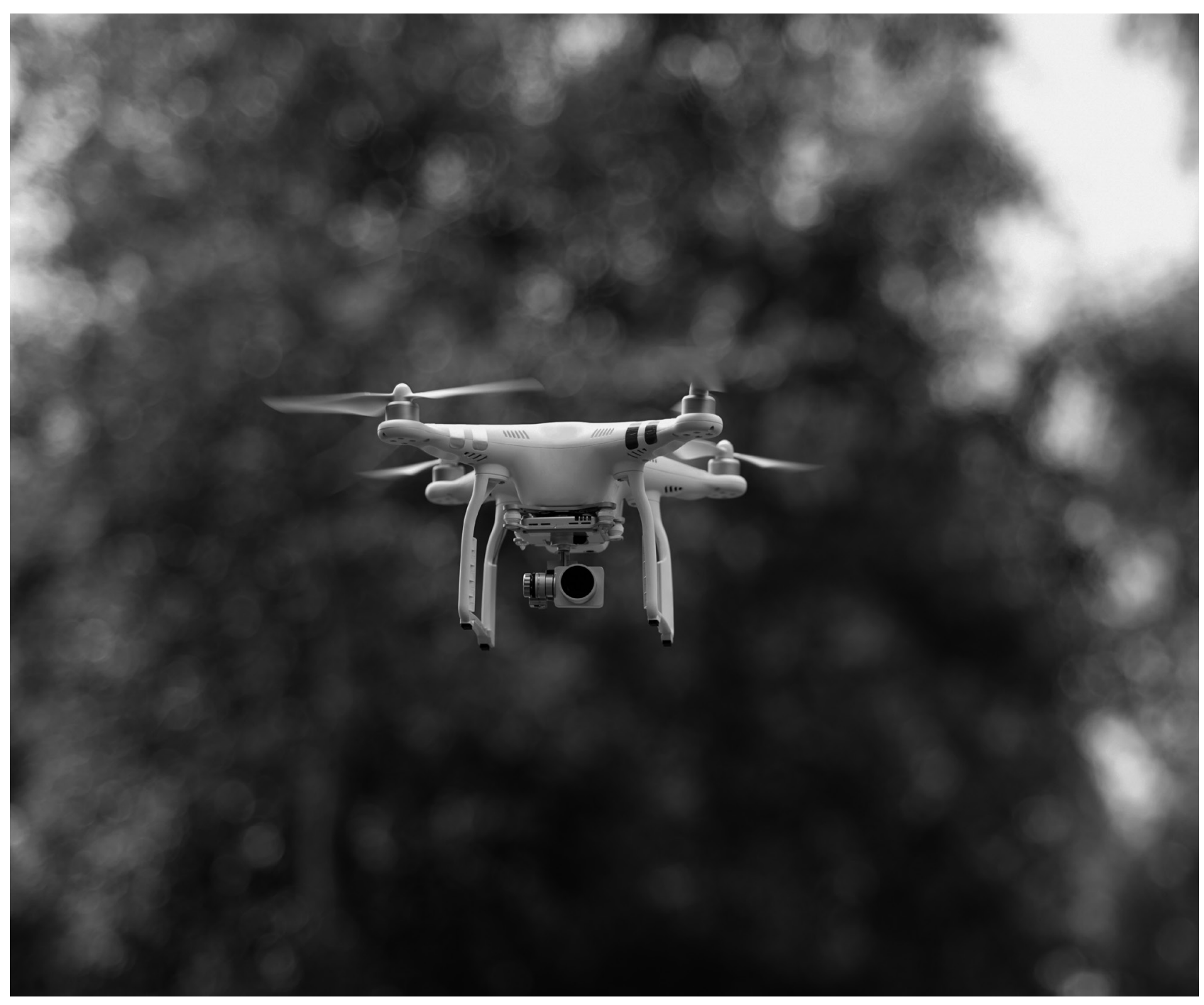




\title{
Control robusto QFT para estabilización en cuadricóptero utilizando técnicas de prototipado rápido ${ }^{1}$
}

\author{
QFT robust control for stabilization in quadrotor based \\ on rapid prototyping techniques
}

\section{Rafael Augusto Núñez Rodríguez², Fabio Alfonso González³ , Eder Fabián García Castro4, Alfredo Gómez Delgado5}

Artículo recibido en octubre de 2016; artículo aceptado en junio de 2017.

Este artículo puede compartirse bajo la Licencia Creative Commons Atribución-No Comercial-Compartir Igual 2.0 Genérica

y se referencia usando el siguiente formato: Núñez, R., González, F., García, E. \& Gómez, A. (2018). Control robusto QFT

para estabilización en cuadricóptero utilizando técnicas de prototipado rápido. I+D Revista de Investigaciones, 11(1), 7-16.

DOI: https://doi.org/10.33304/revinv.v11n1-2018001

\section{Resumen}

Se presenta la implementación de un control robusto basado en la teoría de realimentación cuantitativa, QFT por su sigla en inglés, para un vehículo aéreo no tripulado, tipo cuadricóptero, equipado con sensores inerciales que determinan el ángulo roll y pitch. Se ilustra la metodología que sigue el diseño de este tipo de control, el cual se considera tiene la posibilidad de estabilizar una planta sin importar la presencia de las incertidumbres que otorga el modelo matemático y las perturbaciones externas a partir de la cuantificación de especificaciones de desempeño. Se implementó el controlador utilizando técnicas de prototipado rápido, proceso que hace referencia a técnicas software y hardware requeridas para disminuir los tiempos de desarrollo y la puesta en marcha de sistemas de control, usando un alto nivel de abstracción en la programación. En el proceso de validación del controlador del cuadricóptero se dispuso de un entorno controlado que se sometió a diversas pruebas, obteniendo tiempos de asentamiento rápidos frente a perturbaciones externas, manteniendo la estabilidad robusta del sistema.

Palabras clave: cuadricóptero, estabilidad robusta, IMU, prototipado rápido, QFT.

1. Artículo de investigación de enfoque cuantitativo, resultado de un proyecto de investigación culminado, perteneciente al área de Ingeniería Electrónica, subárea de Control, desarrollado en el Grupo de Investigación de Control Avanzado -Gicav-, fue financiado por las Unidades Tecnológicas de Santander de la ciudad de Bucaramanga (Colombia). Dirección: Calle de Los Estudiantes n. 9 - 82, Ciudadela Real de Minas, PBX 6917700. Fecha de inicio: febrero 2016, Fecha de terminación: noviembre 2016.

2. Ingeniero Electrónico, Unidades Tecnológicas de Santander. Especialista en Control e Instrumentación Industrial, Universidad Pontificia Bolivariana. Docente - investigador del grupo Gicav. Unidades Tecnológicas de Santander de la ciudad de Bucaramanga (Colombia). Dirección: Calle de Los Estudiantes n. 9 - 82, Ciudadela Real de Minas, PBX 6917700. ORCID ID: https://orcid.org/0000-0001-6775-776X. Correo electrónico institucional: rrodriguez@correo.uts.edu.co.

3. Ingeniero Electricista, Universidad Industrial de Santander. Magíster en Potencia Eléctrica, Universidad Industrial de Santander. Docente - investigador del grupo Gicav. Unidades Tecnológicas de Santander de la ciudad de Bucaramanga (Colombia). Dirección: Calle de Los Estudiantes n. 9 - 82, Ciudadela Real de Minas, PBX 6917700. ORCID ID: https://orcid.org/0000-0001-8449-8683. Correo electrónico institucional: fagonzalez@correo.uts.edu.co.

4. Ingeniero Electrónico, Unidades Tecnológicas de Santander de la ciudad de Bucaramanga (Colombia): Calle de Los Estudiantes n.॰ 9-82 Ciudadela Real de Minas, PBX 6917700. ORCID ID: https://orcid.org/0000-0002-4179-2636. Correo electrónico eder-fab@hotmail.com.

5. Ingeniero Electrónico, Unidades Tecnológicas de Santander de la ciudad de Bucaramanga (Colombia). Dirección: Calle de Los Estudiantes n. 9 - 82, Ciudadela Real de Minas, PBX 6917700. ORCID ID: https://orcid.org/0000-0002-6248-1351. Correo electrónico alfregomezdelga@hotmail.com. 


\begin{abstract}
The implementation of a robust control based on the theory of quantitative feedback, QFT for its acronym in English, for an unmanned aerial vehicle, quadrocopters type is presented, it is equipped with inertial sensors that determine the roll and pitch angle. The methodology that follows the design of this type of control is illustrated, which is considered to have the possibility of stabilizing a plant regardless of the presence of uncertainties given by the mathematical model and external perturbations from the quantification of performance specifications. The controller was implemented using rapid prototyping techniques, a process that refers to software and hardware techniques required to reduce development times and the start-up of control systems, using a high level of abstraction in programming. In the process of validation of the quadrocopters controller, a controlled environment was available, which was subjected to various tests, obtaining fast settling times against external disturbances, maintaining the robust stability of the system.
\end{abstract}

Keywords: quadrocopters, robust stability, IMU, rapid prototyping, QFT.

\section{Introducción}

En las últimas décadas, el uso del cuadricóptero ha aumentado debido a los diferentes usos y proyectos de ingeniería que utilizan estas aeronaves. Diversos investigadores (Alcalá, 2014; Carmona, 2013) han propuesto controlar un artefacto de esta naturaleza a través de Wi-Fi, usando un dispositivo móvil. Por otro lado, se encuentran simulaciones del comportamiento dinámico de un cuadricóptero, el control y las pruebas de rendimiento realizadas a este, y visualizadas en un entorno de realidad virtual (Rodríguez \& Jiménez, 2015). De igual forma, se han implementado sistemas de control para la estabilidad y orientación del cuadricóptero basados en una estructura en cascada que utiliza un control PID por ganancia programada y otro control PID autosintonizado por lógica difusa (Ortiz \& Pulla, 2014); otros autores han empleado la metodología de la ingeniería concurrente para definir por completo el problema de la maniobrabilidad. Finalmente, se evidencia el uso de la técnica QFT para seguimiento de referencias de altitud (Otero, 2012), conocida como Teoría de Realimentación Cuantitativa (Quantitative Feedback Theory) (Chen \& Ballance, 1999), una técnica de diseño en el dominio de la frecuencia para sistemas donde la planta presenta incertidumbres y se ve afectada por perturbaciones (Horowitz, 1959; Mansilla, 2007). Se presentan aquí los resultados de la implementación de un controlador robusto QFT en un cuadricóptero montado sobre una estructura de dos (2) grados de libertad, donde se busca la estabilidad de la inclinación y el rechazo a perturbaciones a la salida (Torres-Barreto \& Muñoz-Molina, 2014).

\section{Modelo matemático}

El modelo matemático se obtuvo a través de la dinámica del cuadricóptero, la cual relaciona el torque generado por la fuerza de empuje de los motores Sunnysky $980 \mathrm{Kv}$ y el ángulo de inclinación, medido por el sensor inercial MPU6050, que contiene un acelerómetro y un giroscopio. Se utilizó la configuración en cruz, que consiste en dos motores por eje utilizando una estructura F450, acoplada sobre una estructura de la cual se suspende el cuadricóptero (Arango \& Torres, 2013).

La dinámica se puede expresar mediante la Ecuación (1) (Jaramillo \& Gómez, 2013); de este modo, el ángulo de inclinación $\phi(x)$ depende de la diferencia de empuje de los motores 4 y 2 denominado $\Omega$ x, y el ángulo de inclinación $\theta(y)$ depende de la diferencia de empuje de los motores 1 y 3 denominado $\Omega$ y como se aprecia en la Figura 1.

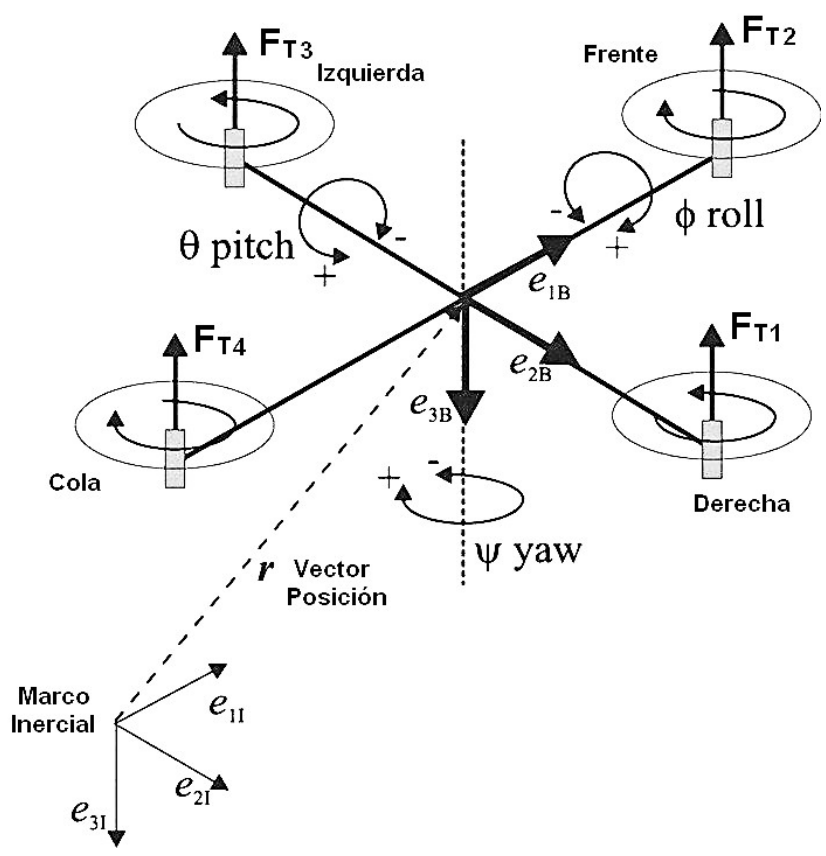

Figura 1. Dinámica del rotacional. Fuente: Bolandi et al. (2013). 


$$
T=\left[\begin{array}{c}
\tau \phi \\
\tau \theta
\end{array}\right]=\left[\begin{array}{l}
l\left(F_{T 4}-F_{T 2}\right) \\
l\left(F_{T 1}-F_{T 3}\right)
\end{array}\right]
$$

Siendo I la distancia desde el centro de gravedad hasta el centro del motor y $F_{-} T 1, F_{-} T 2, F_{-} T 3, F_{-} T 4$ las fuerzas de empuje de cada motor; la Ecuación (1) es la base de la salida, la cual relaciona la segunda derivada de la inclinación con el torque $\tau$ y la inercia $I \_x x, I$ yy ejercida por el eje como se muestra en la Ecuación (2).

$$
\dot{x}=\left\{\begin{array}{l}
\ddot{\phi}=\tau_{\phi} I_{x x}{ }^{-1} \\
\ddot{\theta}=\tau_{\theta} I_{y y}{ }^{-1}
\end{array}\right.
$$

Para obtener la planta en el dominio de la frecuencia se utiliza la transformada de Laplace, cuyo resultado se indica en las Ecuaciones (3) y (4).

$$
\begin{aligned}
& H \emptyset=\frac{\emptyset(s)}{\Omega x(s)}=\frac{l}{I x x s^{2}} \\
& H \theta=\frac{\theta(s)}{\Omega y(s)}=\frac{l}{I y y s^{2}}
\end{aligned}
$$

De lo anterior se concluye que cada eje del cuadricóptero es un sistema con doble integrador, cuyas incertidumbres para el proceso son la distancia desde el centro del motor hasta el centro de masa denominada I y los momentos de inercia de los ejes Ixx,lyy.

\section{Diseño del controlador QFT}

En el diseño de un controlador robusto QFT para un sistema con incertidumbre paramétrica es importante definir el espacio de incertidumbre del sistema, cuantificar las especificaciones de desempeño, calcular los contornos, diseñar el controlador y analizar su respuesta (Garcia-Sanz, Mauch \& Philippe, 2012). La Figura 2 ilustra los pasos que metodológicamente se siguen para diseñar un controlador.

\section{Espacio de incertidumbres}

Con base en los datos experimentales del cuadricóptero se puede establecer una variación en los parámetros del convertidor alrededor del $\pm 50 \%$, para conformar un intervalo de incertidumbre para cada uno de los parámetros que afectan el punto de operación del sistema. El centro de masa tiene una variación tal que $\Delta \mathrm{l}=[0,0029 \mathrm{~m} 0.0087 \mathrm{~m}]$ I E R, y el momento de inercia tiene una variación tal que $\Delta l=\left[0.227 \mathrm{~kg}^{*} \mathrm{~m} 2 \quad 0,228\right.$ $\mathrm{kg}^{*} \mathrm{~m} 2$ ] I E R, lo cual permite generar una familia de plantas evaluadas ante un conjunto de frecuencias de interés entre $0,01 \mathrm{rad} / \mathrm{s}$ a $10 \mathrm{rad} / \mathrm{s}$, obteniendo para cada frecuencia una representación en fase $\left[^{\circ}\right]$ y magnitud [dB] del conjunto de plantas sobre el diagrama de Nichols (Houpis, Rasmussen \& Garcia-Sanz, 2006). En el espacio de incertidumbre se tiene en cuenta, además, un tiempo muerto que corresponde al tiempo de respuesta del sensor y al periodo de muestreo del sistema, que se fijó en $0,0215 \mathrm{~S}$.

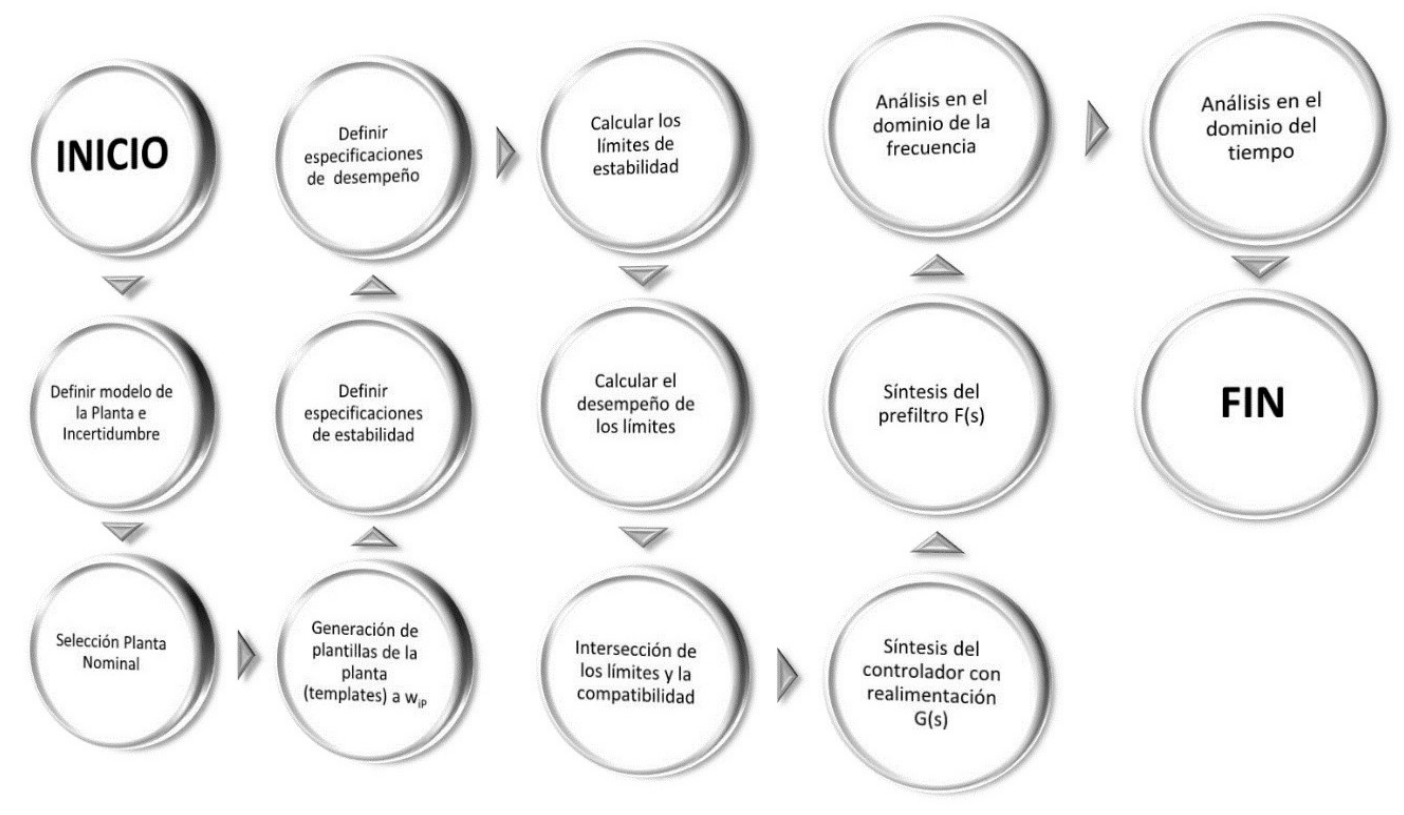

Figura 2.Pasos para el desarrollo del controlador mediante teoría de realimentación cuantitativa QFT. Fuente: Garcia-Sanz (2017). 


\section{Especificaciones de desempeño}

Definida la planta y generadas las plantillas, se introducen las especificaciones de desempeño de estabilidad robusta según Ecuación (5), y el rechazo de perturbaciones a la salida del sistema, dado que el vehículo se somete a cambios del punto de operación y cambios en la inclinación de su eje de referencia. Para la estabilidad robusta se define un margen de fase de $45^{\circ}$ (Biernson, 1988) y un margen de ganancia de $5 \mathrm{~dB}$, que implica un $\delta \_u=1,3$. La sensibilidad complementaria permite establecer condiciones de estabilidad robusta y limitaciones del ancho de banda del sistema en lazo cerrado; por tanto, la especificación de rechazo de perturbación a la salida definida en la Ecuación (6) se cuantifica a partir de la función de transferencia $\delta \_s$ definida en la Ecuación (7), lo que permite al controlador responder más agresivamente frente a las perturbaciones de baja frecuencia que son amplificadas por el sistema (D’Azzo \& Houpis, 2013).

$$
\begin{gathered}
\left|\frac{L(j \omega)}{1+L(j \omega)}\right|<\delta_{u}(j \omega) \\
\left|\frac{1}{1+L(j \omega)}\right|<\delta_{s}(j \omega) \\
\delta_{s}(s)=\frac{40 s+0.1}{0.01 s+20}
\end{gathered}
$$

\section{Contornos del controlador}

A partir de las especificaciones de desempeño del controlador de las Ecuaciones (5) y (6) y los parámetros $\delta \_u(\omega)$ y $\delta \_S(\omega)$, se logra un $L(j \omega)$ tal que cumpla las desigualdades allí establecidas, donde $L(j \omega)=G(j \omega) P(j \omega)$. Así, el problema de control se centra en conformar un único controlador $\mathrm{G}(\mathrm{j} \omega)$ que cumpla con todas las de control se plantea una inecuación cuadrática por cada especificación de desempeño, como se indica en las Ecuaciones (8) y (9). Este sistema de ecuaciones se resuelve a partir de métodos iterativos que se estructuran en un algoritmo (Chait \& Yaniv, 1993), donde g equivale a la representación polar del controlador y $p$ representa la forma polar de la planta nominal del lazo L_o.

Cada raíz del sistema de inecuaciones cuadráticas representa un lugar en el diagrama de Nichols para cada par fase $\left[^{\circ}\right]$ y magnitud $[\mathrm{dB}]$, para las $\mathrm{n}$ frecuencias de interés y la planta nominal, donde se generan $\mathrm{n}$ cantidad de puntos, los cuales representan los contornos de cada especificación, de manera que el único controlador gejo que cumple con todas las especificaciones de desempeño es aquel que consiga llevar la función del lazo $L$ encima de los intercepto de todos los contornos de cada especificación (Gil-Martínez \& García-Sanz, 2003).

$$
\begin{aligned}
& p^{2}\left(1-\frac{1}{\delta_{u}^{2}}\right) g^{2}+2 p \cos (\varnothing+\theta) g \geq 0 \\
& p^{2} g^{2}+2 p \cos (\varnothing+\theta) g+\left(1-\frac{1}{\delta_{s}^{2}}\right) \geq 0
\end{aligned}
$$

Loop-shapping del controlador QFT. Una vez generados los contornos mediante las especificaciones, se diseñó el controlador por medio de la técnica "loop-shaping", que consiste en agregar polos y ceros que modifican el diagrama de Nichols Chart de la planta. Se deben cumplir los contornos generados por las especificaciones dadas anteriormente para cada frecuencia de interés (GilMartínez, 2008), ubicándolos por encima y a su derecha; esto se aprecia en la Figura 3. Se obtuvo entonces la función de transferencia del controlador, expresada en la Ecuación (10) y que se implementó para su validación.

$$
\boldsymbol{Q F T}(\boldsymbol{s})=\frac{20.73 s^{2}+56.94 s+3.412}{0.001347 s^{2}+0.8392 s+1}
$$

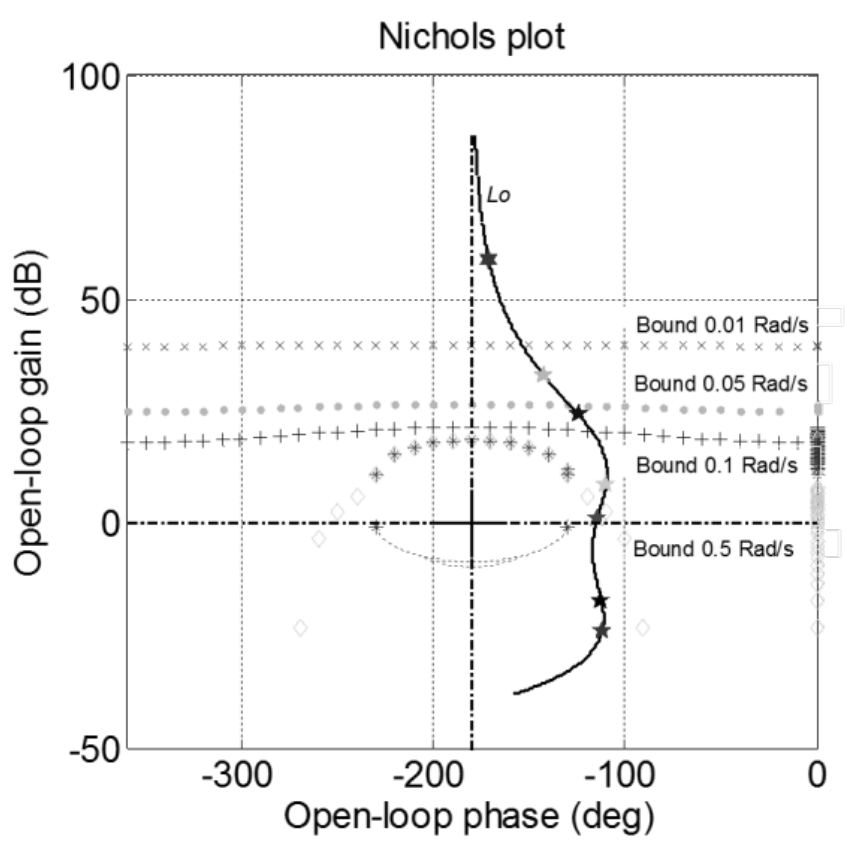

Figura 3. Controlador QFT sintetizado mediante loopshaping. Fuente: Autores. 
Cuando se trata de estabilizar ángulos de inclinación en cuadricópteros para compensar el desequilibrio propio del sistema (Benjumea, 2011), es recomendable adicionar otro lazo de control, ya que este suele no responder como se espera a modificaciones de sus variables. Por esta razón, se implementó un controlador proporcional en paralelo que corrija el ángulo mientras que el controlador QFT se encarga de las variaciones de este con respecto al tiempo. Para el eje de $\phi$ se determinó que la constante proporcional equivale a 0,09 y para el eje $\theta$ toma un valor de 0,1 .

\section{Resultados}

En la Figura 4 se observa el esquema del controlador QFT desacoplado para los ejes del vehículo aéreo, donde cada motor recibe una constante de empuje que mantiene los motores encendidos cuando el cuadricóptero se encuentra estable, al igual que la señal del controlador proporcional y la señal de control del controlador QFT. Los lazos de realimentación se establecen a partir del sensor inercial de cada eje, al igual que la velocidad angular de giro.

Utilizando la Toolbox Embedded Coder de Simulink de Matlab ${ }^{\oplus}$, se configuró la IMU mediante comunicación I2C; se parametrizaron las sensibilidades del acelerómetro a $4 \mathrm{~g}$ y el giroscopio a $250 \%$ y se habilitó un filtro pasabajos con frecuencia de corte de $5 \mathrm{~Hz}$ que mitiga el ruido causado por las vibraciones generadas por los motores. Los ángulos de inclinación se determinaron con el acelerómetro mediante una función trigonométrica tal y como se muestra en las Ecuaciones (11) y (12) (Vela, 2013).

$$
\begin{aligned}
& \emptyset=\operatorname{atan} 2 \frac{a c c_{z}}{a c c_{x}} \\
& \theta=\operatorname{atan} 2 \frac{a c c_{z}}{a c c_{y}}
\end{aligned}
$$

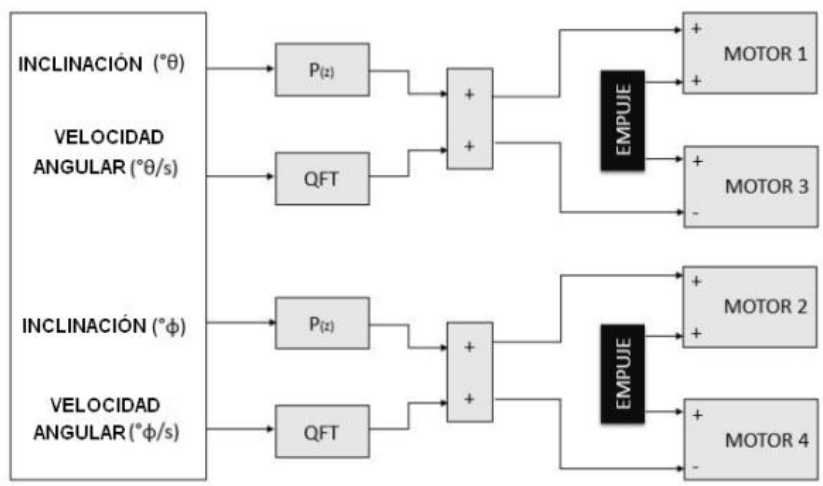

Figura 4. Representación del sistema implementado. Fuente: Autores.
Se empleó un filtro complementario que fusiona las medidas del acelerómetro y el giroscopio dando una mejor fiabilidad en la medición (Gaydou, Redolfi \& Henze, 2011), indispensable para este tipo de sistemas vulnerables al ruido; la estructura de este filtro se ilustra en la Figura 5. Se agregó un filtro Kalman propio de Simulink para mejorar la señal de los sensores inerciales (Pozo, Sotomayor, Rosero \& Morales, 2014), que da resultados excelentes para este tipo de sistemas ya que es un filtro robusto en tanto toma en cuenta la covarianza del proceso y la covarianza del ruido.

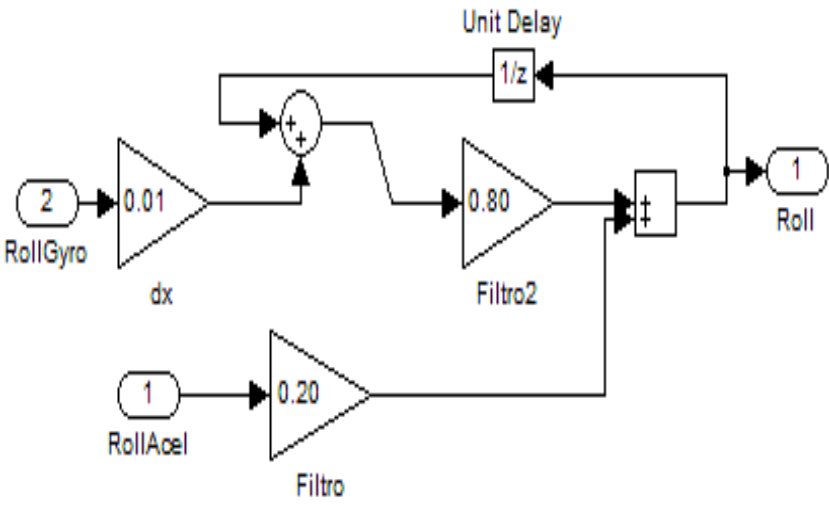

Figura 5. Filtro complementario. Fuente: Autores.

Para validar la respuesta del controlador QFT se realizó la prueba en estado estable de los dos ejes, como se aprecia en la Figura 6 y la Figura 7, donde se observa la oscilación del ángulo de los ejes en función de la referencia, que para este caso se fijó en $0^{\circ}$. Se observa que las variaciones del ángulo son inferiores \pm a sobre el eje de referencia, desviación baja, por lo que el cuadricóptero se mantiene estabilizado en este punto de operación.

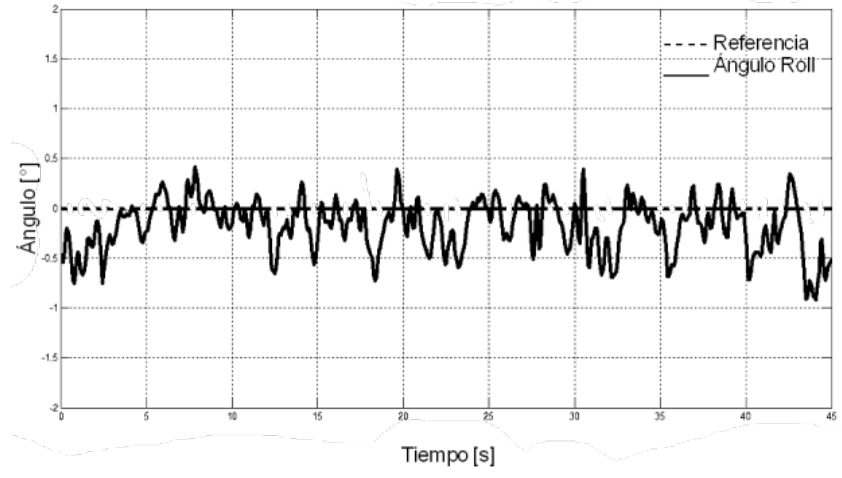

Figura 6. Error en estado estable para ángulo roll. Fuente: Autores. 


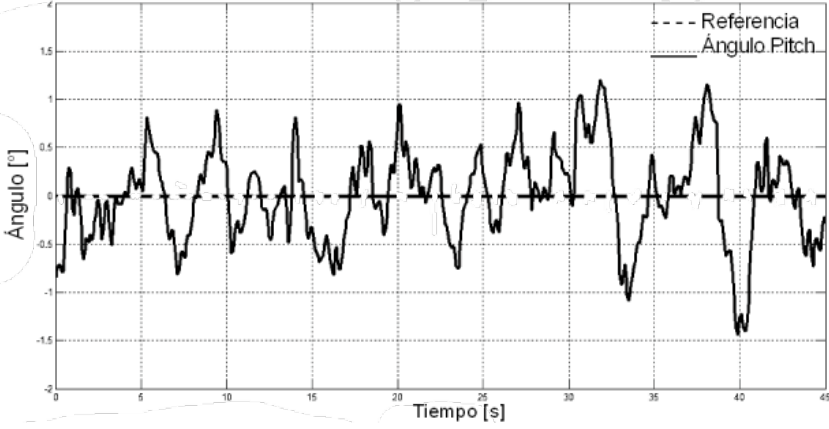

Figura 7. Error en estado estable para ángulo pitch. Fuente: Autores.
La respuesta de cada controlador cuando el cuadricóptero se encuentra estabilizado se muestra en la Figura 8 para el eje roll y en la Figura 9 para el eje pitch. La gráfica superior izquierda de la Figura 8 muestra el ángulo de inclinación del eje en grados; la superior derecha muestra la velocidad angular del eje; la inferior izquierda muestra la señal de control proporcional, y la inferior derecha muestra la señal del control QFT para el ángulo roll.

La gráfica superior izquierda de la Figura 9 muestra el ángulo de inclinación del eje en grados; la gráfica superior derecha muestra la velocidad angular del eje; la inferior izquierda muestra la señal de control proporcional, y la gráfica inferior derecha muestra la señal del control
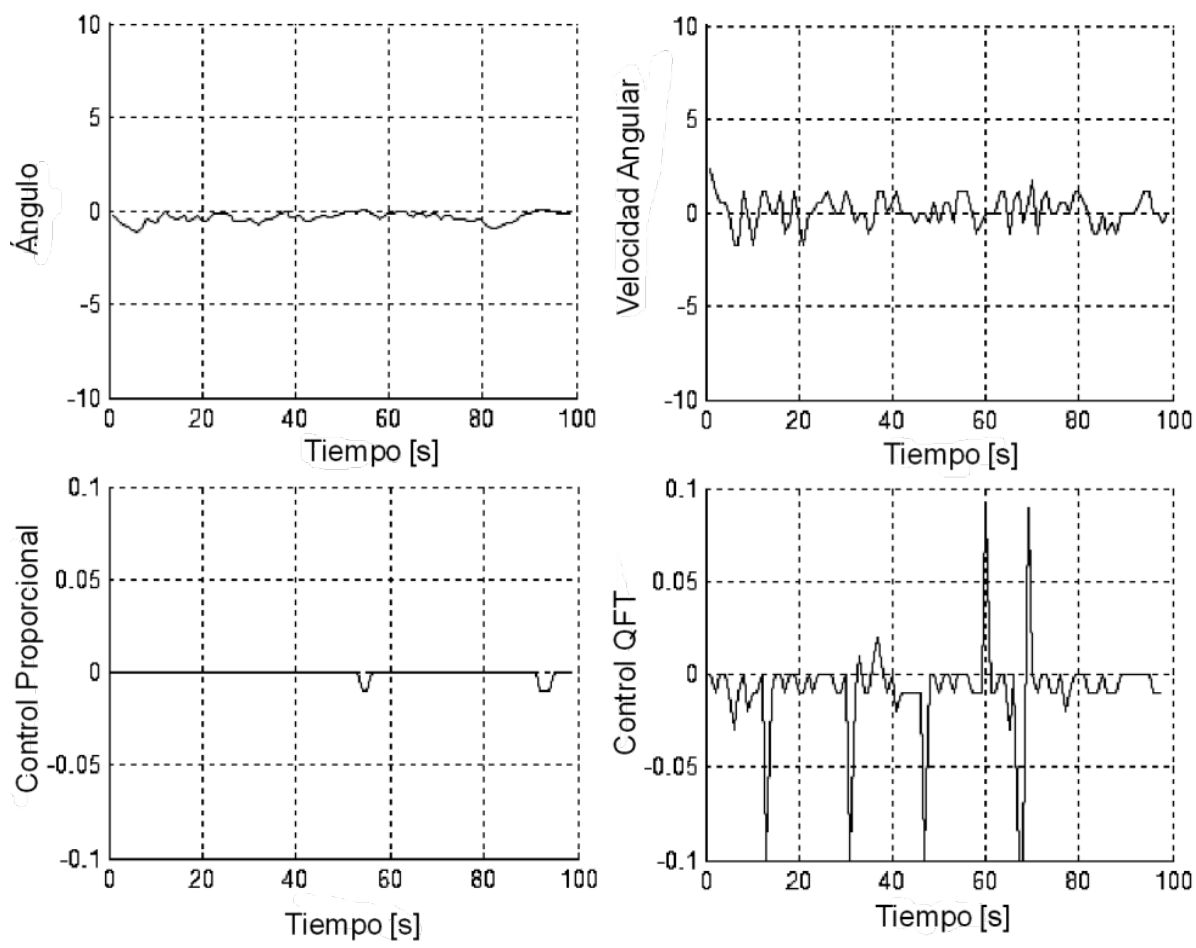

Figura 8. Respuesta del controlador ángulo roll. Fuente: Autores.

QFT para el ángulo pitch. Al estar estabilizado el eje, las señales de control son relativamente pequeñas, no superiores a 0,1 , debido a que no hay perturbaciones $y$ el eje se encuentra estabilizado. El controlador proporcional entrega valores aún más pequeños, que ayudan a corregir el error del ángulo de inclinación.

Para validar la respuesta del controlador frente a perturbaciones externas se sometió el sistema a un cambio de inclinación en el plano de referencia del vehículo y se soltó hasta regresar a $0^{\circ}$ nuevamente.
Esto permite observar el tiempo que tarda el controlador en mitigar los efectos de esta perturbación en cuanto a la estabilización de los ejes del cuadricóptero. El tiempo de establecimiento obtenido es aproximadamente de 2 a $4 \mathrm{~s}$ para una inclinación de $5^{\circ}$. De igual forma, se observa la respuesta del controlador QFT y la respuesta del lazo de control proporcional. La gráfica superior izquierda de la Figura 10 muestra el ángulo de inclinación del eje en grados; la gráfica superior derecha muestra la velocidad angular del eje; la inferior izquierda muestra la señal de control proporcional, y la gráfica inferior derecha muestra la señal del control QFT para la perturbación en el ángulo roll. 

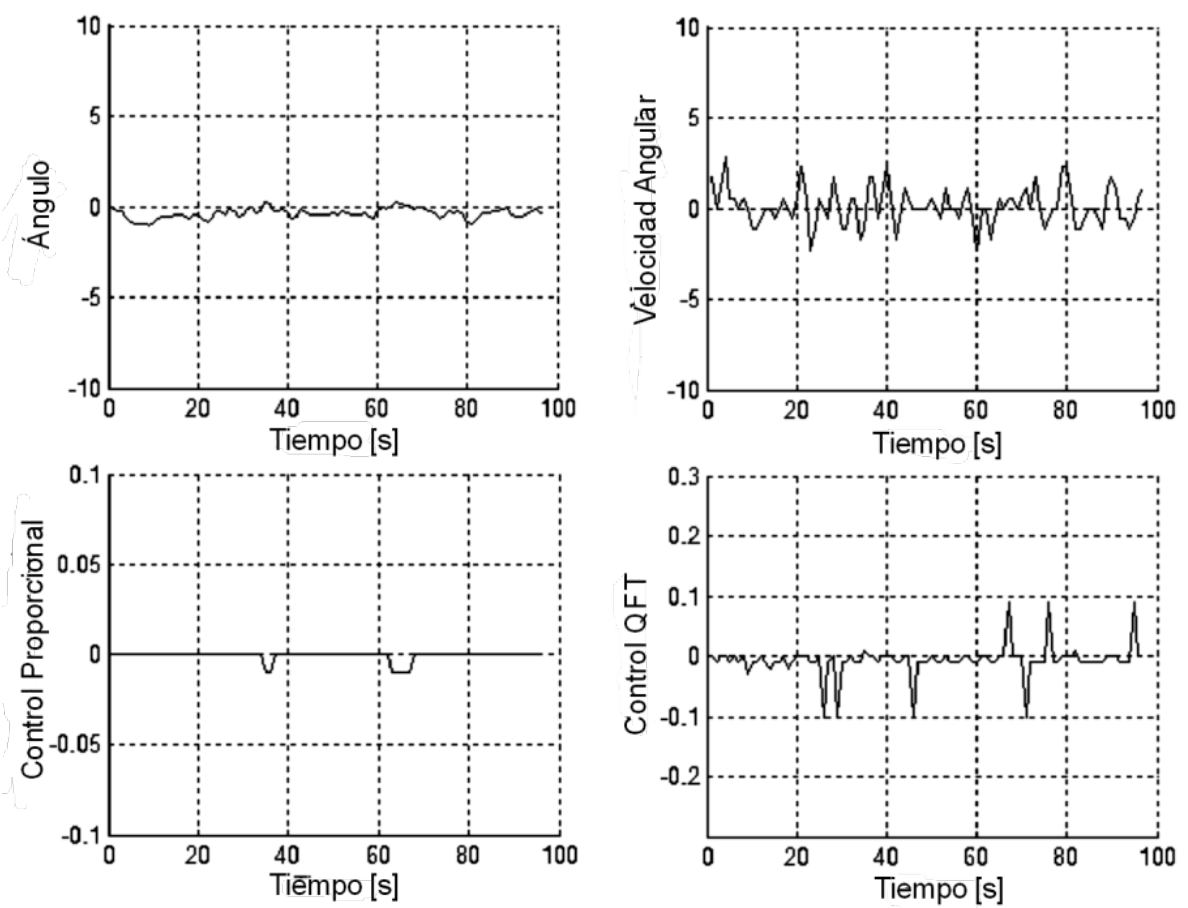

Figura 9. Respuesta del controlador ángulo pitch. Fuente: Autores.
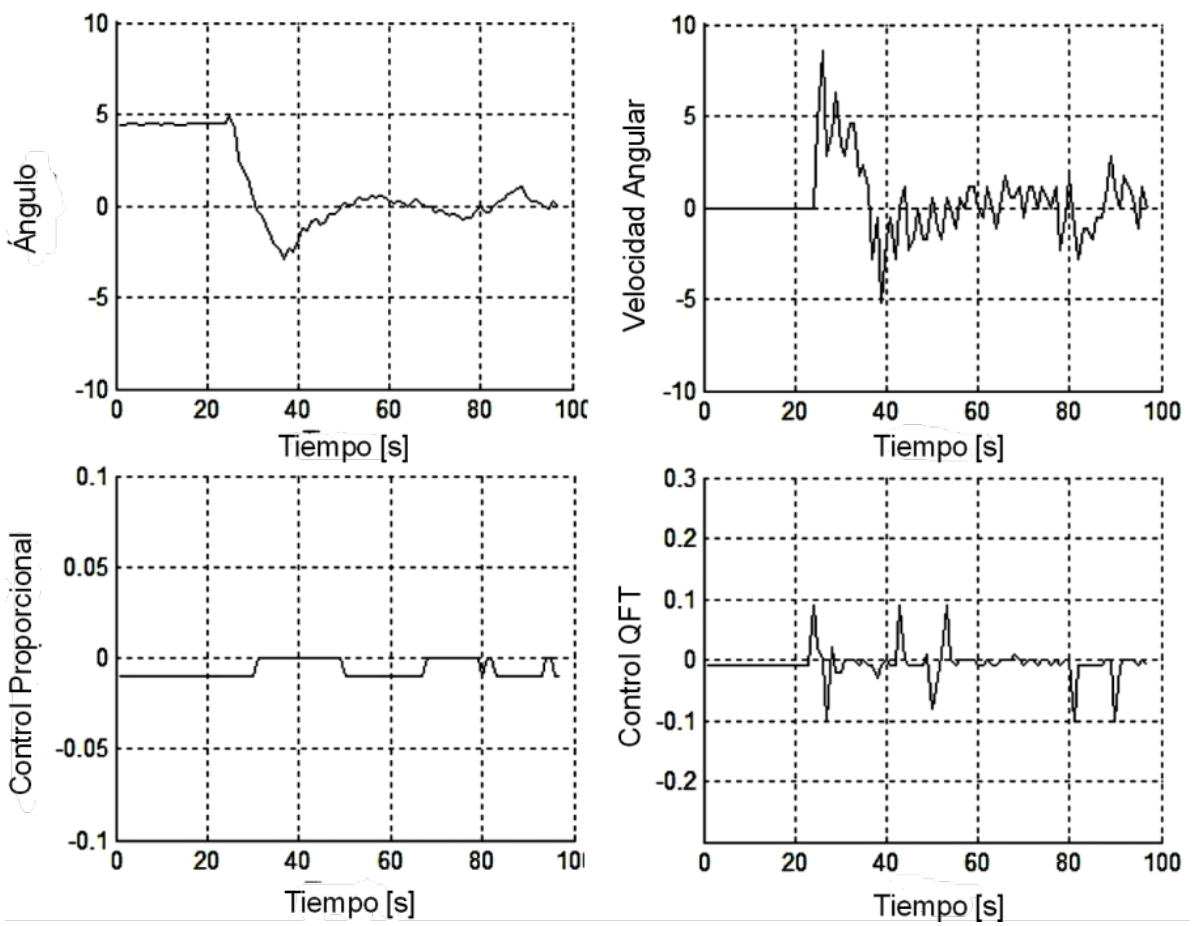

Figura 10. Análisis de perturbación ángulo roll. Fuente: Autores. 
La gráfica superior izquierda de la Figura 11 muestra el ángulo de inclinación del eje en grados; la gráfica superior derecha muestra la velocidad angular del eje; la inferiorizquierda muestra la señal de control proporcional, y la gráfica inferior derecha muestra la señal del control QFT para la perturbación del ángulo pitch.
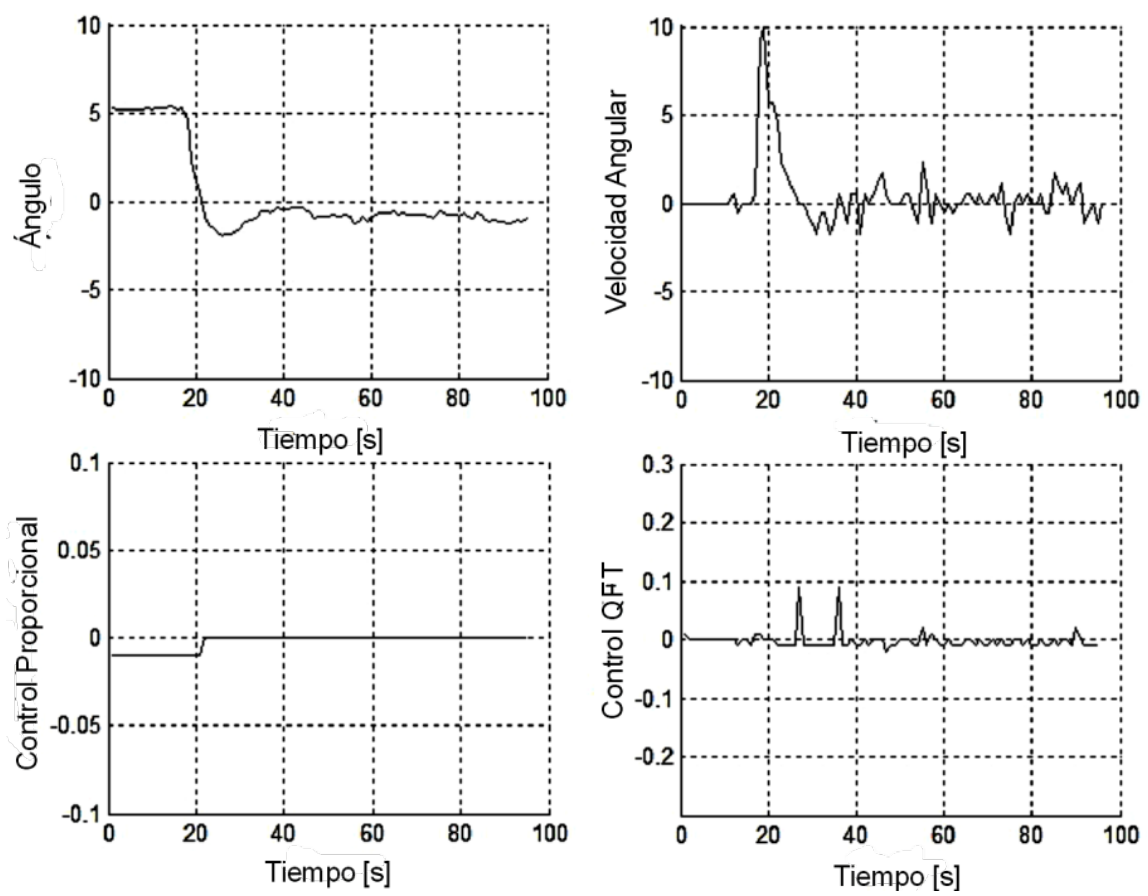

Figura 11. Análisis de perturbación ángulo pitch. Fuente: Autores.

Se observa cómo reaccionan los controladores ante un escalón y se verifica que el controlador QFT sea el que genere la señal de control para rechazar esta perturbación, la cual se determina por la velocidad angular, mientras que el controlador proporcional genera una señal de control cuando el ángulo es diferente de $0^{\circ}$, dando estabilidad al sistema y teniendo un tiempo de estabilización de entre 3 y 4 segundos.

\section{Conclusiones}

Para evitar la generación de errores es de gran importancia elegir una estructura de pruebas que no modifique o agregue propiedades físicas al sistema, como es el caso de mover el centro de masa del centro de rotación.

Debido a la intensidad del ruido en la medición del ánguloy la velocidad angular, no se logró obtener un valor confiable con una técnica de filtrado convencional, por esto fue necesario utilizar, además del filtro incorporado en la IMU, un filtro complementario en la programación que permitiera obtener un valor confiable. Adicionalmente, se utilizó un filtro Kalman para suavizar la señal que generan los sensores.

El prototipado rápido que ofrece Mathworks automatiza el trabajo de desarrollo de modelos y algoritmos de control sobre hardware para ejecutarlos en tiempo real, permitiendo centrar la atención en la evaluación del desempeño de los modelos y la mejora de estos, cumpliendo con la expectativa que se proyectó sobre este en el trabajo realizado sobre el cuadricóptero.

El controlador diseñado presentó una rápida respuesta ante perturbaciones externas, estabilizando el sistema en un tiempo de asentamiento de 2 a 5 segundos dependiendo de la perturbación, que se refleja, desde el punto de vista físico, en un error en estado estable de $\pm 1.5^{\circ}$ de inclinación en cada eje del cuadricóptero.

El controlador diseñado que controla dos ejes simultáneamente presentó menor error en estado estable para el eje $Y$ (pitch) de $\pm 1^{\circ}$ de inclinación, en comparación con el eje $x\left(\right.$ roll), que presentó $\pm 1.5^{\circ}$.

\section{Referencias}

Alcalá, E. (2014). Desarrollo e implementación de un cuadricóptero. Universidad de Zaragoza. Recuperado de https://zaguan.unizar.es/record/14367? ln=es 
Arango, J. D. \& Torres, V. (2013). Diseño incluyente desde el proceso comunicativo usuario-dispositivos para divergencia funcional locomotriz. I+D Revista de Investigaciones, 1(1), 53-59

Benjumea, J. (2011). Control de trayectorias de un vehículo aéreo no tripulado de alas rotatorias tipo quadrotor en ambientes exteriores.

Biernson, G. A. (1988). Feedback System Design, Volume 1, Principles Of Feedback Control By George A. Biernson. Wiley-Interscience.

Bolandi, H., Rezaei, M., Mohsenipour, R., Nemati, H. \& Smailzadeh, S. M. (2013). Attitude Control of a Quadrotor with Optimized PID Controller. Intelligent Control and Automation, 4(3), 335-342. Recuperado de $\mathrm{http}: / /$ doi. org/10.4236/ica.2013.43039

Carmona, J. (2013). Diseño de un sistema de control para un cuadricóptero: Universidad Carlos III de Madrid.

Chait, Y. \& Yaniv, O. (1993). Multi-input/single-output computer-aided control design using the quantitative feedback theory. International Journal of Robust and Nonlinear Control, 3(1), 47-54. Recuperado de https://doi.org/10.1002/rnc.4590030103

Chen, W. \& Ballance, D. J. (1999). Plant Template Generation for Uncertain Plants in Quantitative Feedback Theory. Journal of dynamic systems, measurement, and control, 121(3), 358-364.

D’Azzo, J. J. \& Houpis, C. H. (2013). Linear Control System Analysis and Design with MATLAB ${ }^{\circledR}$.

Garcia-Sanz, M., Mauch, A. \& Philippe, C. (2012). Appendix FQFT control toolbox (QFTCT) user's guide.

Garcia-Sanz, M. (2017). Robust control engineering: practical QFT solutions. Boca Raton: CRC Press.

Gaydou, D., Redolfi, J. \& Henze, A. (2011). Filtro complementario para estimacion de actitud aplicado al controlador embebido de un cuatrirrotor. Recuperado de http://proyectos.ciii.frc.utn.edu.ar/cuadricoptero/ export/9ed95816c90cc7d83e32fd2e13b032dc515c0d7a/documentacion/informe_final/paper_case.pdf

Gil-Martínez, M. M. \& García-Sanz, M. (2003). Simultaneous meeting of robust control specifications in QFT. International Journal of Robust and Nonlinear Control, 13(7), 643-656.

Gil-Martínez, M. (2008). Síntesis de Controladores robustos mediante el análisis de la compatibilidad de especificaciones e incertidumbre: Universidad de la Rioja.

Horowitz, I. (1959). Fundamental theory of automatic linear feedback control systems. IRE Transactions on Automatic Control, 4(3), 5- 9. Recuperado de http:// doi.org/10.1109/TAC.1959.1104893

Houpis, C. H., Rasmussen, S. J. \& Garcia-Sanz, M. (2006). Quantitative feedback theory: fundamentals and applications. CRC Taylor \& Francis. Recuperado de https:// www.crcpress.com/Quantitative-Feedback-TheoryFundamentals-and-Applications-Second-Edition/Houpis-
Rasmussen-Garcia-Sanz/p/book/9780849333705

Jaramillo, F. \& Gómez, A. (2013). Sistema de control para la estabilidad y orientación de un helicóptero quadrotor.

Mansilla, R. (2007). Control multivariable mediante técnicas QFT: Aplicaciones a Sistemas Navales. (Doctoral dissertation, Universidad Nacional de Educación a Distancia. UNED).

Ortiz, V. G. \& Pulla, P. R. (2014). Diseño y construcción de un cuadricóptero a control remoto. Sangolquí - Ecuador.

Otero, J. (2012). Diseño implementación y prueba de técnicas de control robusto aplicadas a la operación de un UAV (Unmanned Air Vehicle). Recuperado de http:// e-spacio.uned.es:8080/fedora/get/tesisuned:InglnfJlopez/Documento.pdf

Pozo, D., Sotomayor, N., Rosero, J. \& Morales, L. (2014). Medición de ángulos de inclinación por medio de fusión sensorial aplicando filtro de Kalman. Revista EPN, 1(1). Recuperado de http://www. revistapolitecnica.epn.edu.ec/ojs2/index.php/ revista_politecnica2/article/view/144/pdf

Rodríguez, D. L. \& Jiménez, S. E. (2015). Diseño e implementación de una estrategia de control óptimo para simulación de un vehículo aéreo no tripulado: Universidad Distrital "Francisco José de Caldas." Recuperado de http://repository.udistrital.edu. co/ bitstream/11349/4205/1/RodriguezMolinaDavidLeonardo2015.pdf

Torres-Barreto, M. L., \& Muñoz-Molina, L. P. (2014). Macrotendencias en textrónica y objetos inteligentes. I+D Revista de Investigaciones, 4(2), 88-103

Vela, R. (2013). Sistema de detección de movimientos basado en sensores inerciales integrados. 1-4. 